\title{
Argumentation et Analyse du Discours
}

Chateauraynaud, Francis. 2011. Argumenter dans un champ de forces. Essai de balistique sociologique (Paris : Editions Petra, coll. "Pragmatismes »)

\section{Marianne Doury}

\section{(2) OpenEdition}

Journals

Édition électronique

URL : http://journals.openedition.org/aad/1245

DOI : 10.4000/aad. 1245

ISSN : $1565-8961$

Éditeur

Université de Tel-Aviv

Référence électronique

Marianne Doury, «Chateauraynaud, Francis. 2011. Argumenter dans un champ de forces. Essai de balistique sociologique (Paris : Editions Petra, coll. "Pragmatismes ») », Argumentation et Analyse du Discours [En ligne], 8| 2012, mis en ligne le 15 avril 2012, consulté le 21 septembre 2020. URL : http:// journals.openedition.org/aad/1245; DOI : https://doi.org/10.4000/aad.1245

Ce document a été généré automatiquement le 21 septembre 2020.

\section{(i)

Argumentation \& analyse du discours est mis à disposition selon les termes de la licence Creative Commons Attribution - Pas d'Utilisation Commerciale - Pas de Modification 4.0 International. 


\title{
Chateauraynaud, Francis. 2011.
} Argumenter dans un champ de forces. Essai de balistique sociologique (Paris : Editions Petra, coll. « Pragmatismes »)

\author{
Marianne Doury
}

\section{RÉFÉRENCE}

Chateauraynaud, Francis. 2011. Argumenter dans un champ de forces. Essai de balistique sociologique (Paris : Editions Petra, coll. « Pragmatismes »), 477 pages, ISBN :

9782847430394

1 Le présent compte rendu propose un point de vue très particulier sur l'ouvrage de Francis Chateauraynaud, Argumenter dans un champ de forces. En effet, aussi diversifiés que soient les références mobilisées et les rapprochements disciplinaires opérés, la réflexion proposée dans cet essai n'en est pas moins centralement caractérisée comme une sociologie (balistique sociologique, ou sociologie argumentative, mais sociologie quand-même); or, c'est en tant que linguistique intéressée aux questionnements argumentatifs que je l'ai lue, comprise et appréciée. C'est donc un éclairage partiel qui en sera construit ici : il s'agit pour moi d'expliciter ce qui, dans le programme élaboré par Francis Chateauraynaud, peut contribuer à enrichir, et dans une certaine mesure, à renouveler, les études en argumentation, dès lors qu'elles cherchent à rendre compte d'échanges argumentés inscrits dans leur contexte social. 


\section{Une approche descriptive et analytique}

2 L'ouvrage de Chateauraynaud, ancré dans une sociologie pragmatique et réflexive, développe une approche des controverses et des conflits qui privilégie la dimension descriptive et analytique. Le modèle élaboré s'interdit de plaquer sur les objets dont il cherche à rendre compte une vision prophétique ou apologétique, mais «laisse les principes et les valeurs aux acteurs » qui en nourrissent leurs argumentations et positionnements, et les enrichissent par des retours réflexifs plus ou moins sophistiqués. Il déploie une casuistique visant avant tout à accroître l'intelligibilité des objets qu'il se donne en proposant de les formaliser sur un modèle balistique formalisation par principe ouverte, inachevée, perfectible et adaptable aux spécificités des objets traités, mais suffisamment générale pour permettre des rapprochements entre des processus jusque là envisagés isolément. Au fil de l'ouvrage, ce modèle est mis à l'épreuve d'une multitude de dossiers, de l'amiante aux OGM, du nucléaire au poulet à la dioxine en passant par les avions renifleurs ou la régularisation des sans-papiers : la balistique sociologique développée par Chateauraynaud se justifie par le fait qu'elle permet de parler du monde - un monde complexe, souvent sombre, où « des systèmes électriques lâchent, des ponts s'effondrent, des avions s'écrasent, des déchets toxiques se répandent, des ferrys se renversent, des tempêtes ou des ouragans dévastent des régions entières » (333).

3 Le type de questions soulevé par ces études de cas, et auquel cet ouvrage cherche à répondre est : qu'est-ce qui fait qu'une cause « porte » ou pas, qu'elle devienne audible et réussisse à emporter la conviction au-delà de l'échelle locale qui l'a vue émerger, ou qu'au contraire, elle stagne, s'étouffe et soit dégradée en « cause perdue »?

\section{Une sociologie argumentative}

4 Le cadre proposé pour se saisir de ces questions est ce que Chateauraynaud appelle une sociologie argumentative. Si l'étude sociologique des controverses doit nécessairement déployer une dimension argumentative, c'est que les acteurs sont sommés, en diverses circonstances, de produire une performance argumentative, et de faire la preuve d'une double capacité d'invention argumentative et de résistance à la critique. La sociologie des controverses ne peut donc se limiter à restituer les jeux d'acteurs; encore faut-il qu'elle articule ces jeux d'acteurs aux jeux d'arguments qui les doublent dans des arènes diverses. La mise en garde contre un fantasme d'autosuffisance vaut pour les sociologues aussi bien que pour les linguistes qui se soucient d'argumentation. Si les débats et controverses ne se réduisent pas à des rapports de force, leur description n'est pas épuisée non plus par une analyse immanentiste des échanges argumentatifs qui les animent: pour Chateauraynaud, les arguments sont susceptibles de "porter ", c'est-à-dire de modifier les catégories et représentations communes, ce qui interdit à l'analyse du discours ou à la linguistique de les considérer comme leur chasse gardée. L'articulation entre ces effets cognitifs de l'argumentation et leur traduction en termes d'actions, collectives ou individuelles, n'est pas facile à élucider ; pourtant, il faut bien constater que les mobilisations en tout genre s'accompagnent nécessairement de la production de discours argumentés - ce qui permet de voir en ces derniers une condition de leur déploiement. 


\section{Une balistique sociologique}

5 Afin de décrire la trajectoire des causes et des arguments qui les soutiennent, Chateauraynaud met en place un jeu de langage autour du champ lexical de la balistique qui, il le reconnaît lui-même, est susceptible de faire partiellement obstacle à la pleine intelligibilité du modèle élaboré : l'utilisation métaphorique du vocabulaire de la «trajectoire », des « angles de vue », de la « portée », des «bifurcations ", jusqu'à la reproduction $\mathrm{du}$ "modèle pur de la parabole ", génère du flou et une forme d'opacité dans la description d'une réalité déjà en elle-même éminemment complexe. Ce jeu de langage est d'autant plus déroutant qu'il s'articule avec plus ou moins de bonheur avec d'autres fantaisies lexicales, originales ou réappropriées par l'auteur, comme le vocabulaire de la prise et de l'emprise, de la preuve et de l'épreuve, du pli, du champ de forces et des puissances d'expression. Mais, si exigeante, et parfois irritante, qu'elles rendent la lecture d'Argumenter dans un champ de forces, ces variations lexicales, sociologie réflexive oblige, font l'objet d'un retour «méta " conséquent, dans lequel l'auteur déroule les jeux de langage autorisés par une "sociologique balistique », et le potentiel heuristique associé. Et de mon point de vue, ces effets sur le cadrage des processus soumis à l'analyse et les questionnements définis comme centraux par une telle sociologie des controverses compensent largement l'inconfort de lecture.

\section{Une pragmatique des transformations}

6 D'une manière générale, le cadrage balistique élaboré par Chateauraynaud met l'accent sur le mouvement, les transformations, les déplacements, les traductions, évolutions et reformulations des causes et des arguments qui les accompagnent - et contribuent à les définir. Loin d'une vision statique ou déterministe, la balistique sociologique s'inscrit dans une pragmatique des transformations et accorde une attention centrale au travail politique opéré par les acteurs, sans laquelle l'émergence et la trajectoire des causes risquent fort de rester inintelligibles. Plus spécifiquement, la perspective ouverte ici insiste - et c'est, de mon point de vue, un apport crucial aux théories de l'argumentation - sur l'importance de la temporalité dans l'analyse des débats; temporalité propre à la controverse, à son évolution, à ses avatars successifs d'une arène à l'autre sous la pression des épreuves critiques, et qui définit la genèse des arguments ; mais aussi temporalité construite discursivement par les acteurs, afin de conférer un sens et une orientation argumentative aux événements qui jalonnent les différents dossiers (de l'amiante, des OGM, du nucléaire...). Chateauraynaud met au jour le travail des acteurs qui totalisent les "séries" passées, érigent des événements saillants en précédents, et s'appuient sur leur compréhension de ce qui a déjà eu lieu et de ce qui est en train d'advenir pour extrapoler à des visions du futur dont l'orientation argumentative est souvent clairement perceptible, selon qu'elles dépeignent un futur désirable (et ouvrent ainsi la voie, par exemple, à un argument pragmatique par les conséquences positives) ou qu'elles font fonction de repoussoir (et orientent alors davantage vers un argument pragmatique par les conséquences négatives).

Cette importance accordée à la temporalité marque depuis longtemps les travaux de Chateauraynaud, via notamment le registre de l'irréversibilité, identifié dès les années 1990 et «tracé » par le logiciel Prospéro dans divers dossiers, mais elle entre ici dans une résonance heureuse avec l'isotopie balistique. 
8 Par cette prise en compte du caractère évolutif des controverses, sans cesse retravaillées par les acteurs au fil du temps et de la succession des arènes qu'elles traversent, Chateauraynaud entend dépasser le dualisme encore trop souvent de mise entre internalisme - priorité donnée à l'étude des arguments pris pour eux-mêmes - et externalisme - prédominance des jeux d'acteurs et des forces qu'ils composent dans le monde (120) -, aussi bien que l'opposition entre approches « micro » et « macro » (20), montrant comment des événements locaux sont susceptibles d'affecter des causes globales (et inversement). Prenant au sérieux la compétence sociale des acteurs, il met au jour la façon dont ceux-ci "jonglent» incessamment entre différentes échelles d'appréhension des trajectoires des causes qu'ils défendent ou combattent.

Un autre intérêt de la démarche proposée par Argumenter dans un champ de forces est d'élaborer un modèle de description des controverses qui, tout en proposant une configuration susceptible de faire apparaître les dynamiques des processus à l'œuvre (voir le cycle idéal en cinq étapes décrit p. 19), reste ouvert, et fasse la place à l'invention (au sens de l'inventio rhétorique) des acteurs, à leur créativité, à leur capacité à renouveler les répertoires d'arguments disponibles au fil notamment des événements.

\section{Articulation entre sociologie, analyse du discours et argumentation : Risques et bénéfices}

10 Enfin, la tentative d'articulation entre sociologie et argumentation, telle qu'elle est élaborée ici, me semble d'un intérêt majeur pour l'analyse des controverses. Chateauraynaud le montre bien, les arguments ne sont pas des jeux logico-langagiers gratuits ; leur omniprésence dans les échanges sociaux, des conversations quotidiennes aux discours politiques institués, suggère qu'ils répondent à une nécessité sociale ; par ailleurs, ils ont des effets en retour sur les milieux qu'ils traversent, ne serait-ce que par leur capacité à manifester et configurer les accords et désaccords entre acteurs de la controverse. En tant que linguiste intéressée à l'argumentation, je ne peux qu'être séduite par la volonté manifestée par le modèle de balistique sociologique, tel qu'il est présenté ici, de prendre au sérieux les arguments avancés par les acteurs, et de les mettre en regard avec la description des dispositifs, des milieux et des champs de forces qui contraignent leur occurrence et leur formulation. Un tel souci d'interdisciplinarité suppose une gymnastique intellectuelle qui n'est pas de tout repos: la lecture d' Argumenter dans un champ de forces demande au non sociologue un véritable effort pour s'approprier l'univers conceptuel déployé par l'auteur. Symétriquement, l'intégration de considérations langagières et argumentatives dans le cadre d'une sociologie pragmatique et réflexive est une démarche délicate, qui évite difficilement quelques "hoquets" terminologiques ou conceptuels. On regrettera par exemple un emploi souvent indécis de termes comme "argument ", «argumentation » et "énoncé », ou une caractérisation discutable de l'opposition entre l'argumentation telle qu'elle est conçue par Perelman et telle qu'elle est élaborée par Ducrot. De telles libertés avec les travaux en linguistique, analyse du discours ou argumentation témoignent de l'attitude adoptée par Chateauraynaud à leur égard: il ne prétend à aucun moment être, ni devenir, un expert de ces paradigmes, mais les parcourt, avec une irrévérence parfois irritante, mais sans doute nécessaire, afin d'enrichir et reformuler le cadre disciplinaire dans lequel sa réflexion s'enracine, celui d'une sociologique pragmatique et réflexive. 
Ce choix apparaît, au terme de la lecture d'Argumenter dans un champ de forces, indéniablement productif : il en résulte une réflexion très stimulante, qui amène à renouveler les questions de recherche que devrait se poser l'analyste engagé dans l'examen de controverses.

\section{AUTEURS}

\section{MARIANNE DOURY}

CNRS, Laboratoire Communication et Politique 